\title{
A comprehensive review on the nutritional and therapeutical aspects of Stevia rebaudiana Bertoni
}

\begin{abstract}
In late years there has been an increase of scientific evidence that supports the use of Stevia rebaudiana Bertoni ( $S$. rebaudiana) as an alternative to sucrose and artificial sweeteners. In addition to its utility in different industries, S.rebaudiana contains various chemical components with potential benefits for human health such as polyphenols, chlorophylls, carotenoids, among others, which can be extracted for the production of nutraceuticals and functional foods. So far no adverse effects on human health have been proven after ingestion and favorable results have been documented when S.rebaudiana or its derivates are included in treatments against diabetes, certain types of cancer, glucose intolerance, obesity, dental cavities, among others. The purpose of this review is to provide an overview of the clinical, chemical and biochemical literature that presents evidence of the beneficial role of Stevia rebaudiana Bertoni and its metabolites in health promotion properties, as well as its relevance in the pharmaceutical and food industries.
\end{abstract}

Volume 6 Issue 6 - 2019

\author{
Escutia-López Karina Nathalie, Sánchez- \\ Pardo Ma Elena, Mora-Escobedo R \\ National Polytechnic Institute, México
}

Correspondence: Rosalva Mora Escobedo, Escuela Nacional de Ciencias Biológicas, Instituto Politécnico Nacional, Unidad Profesional Adolfo López Mateos, Av. Wilfrido Massieu 399, C.P.07738. Ciudad de México, CDMX, México,

Email rosalmorae@gmail.com

Received: November 15, 2019 | Published: December 06 2019

Keywords: stevia leaves, stevia glycosides, phytochemicals, medicinal uses

Abbreviations: DMBA, 7,12-dimethylbenz [a] anthracene; EFSA, European food safety authority; ADI, admissible daily intake; TPA,12-O-tetradecanoylphorbol-13-acetate.

\section{Stevia rebaudiana Bertoni}

Stevia rebaudiana Bertoni, commonly known as stevia, is a perennial shrub belonging to the Asteraceae family; from the Amambay region, in the northeast of Paraguay; although it has also been related to areas of Brazil and Argentina. It is one of the 950 genera of the Compositae asteraceae. Although Dr. Moisés Santiago Bertoni rediscovered stevia in 1887 , the plant has been used by the Guaraní Indians for hundreds of years. Stevia has a long history of medicinal use in Paraguay and Brazil and, although many of the therapeutic applications of stevia have not been scientifically proven, they must be considered since they have been transmitted for generations for hundreds of years. Now it is known that there are more than 150 species of stevia, but Stevia rebaudiana Bertoni is the only one with important sweetening properties. ${ }^{1}$ Stevia is known mainly for the high content of steviol glycosides in its leaves which, as mentioned above, are used in a wide range of food products as a sweetener without sucrose and calories. In addition to glycosides, stevia leaves contain important phytochemical constituents such as water-soluble flavonoids, alkaloids, chlorophylls and xanthophylls, hydroxycinnamic acids (caffeic acid, chlorogenic acid, etc.), neutral water-soluble oligosaccharides, free sugars, amino acids, lipids, essential oils, proteins and oligo-elements. ${ }^{2}$

\section{Other varieties}

The Stevia genus includes more than 200 species, however, only two of them contain these glycosides, with Stevia rebaudiana Bertoni being the variety with the sweetest compounds (Brandle et al., 1998) Among the varieties we can find the following: $S$. eupatoria, S. lemmonii, S. micrantha, S. ovata var. texana, $S$. plummerae, S. plummerae var. alba, S. rhombifolia, S. salicifolia, $S$. serrata, S. viscida, S. commixta, S. satureiaefilia, S. leptophylla, S. myriadenia, S. ophryphylla, S.selloi, S. nepetifolia, S. oligophylla, S. origanoides and S. triflora. ${ }^{3}$

\section{What steviol glycosides are?}

Steviol glycosides such as stevioside and rebaudioside A are organic molecules that have a sweet taste without caloric content and are present in the plant known as Stevia rebaudiana Bertoni, which has been widely used in the industry to sweet beverages and various foods. Stevioside is a diterpenoid glycoside comprising an aglycone (steviol) and three glucose molecules. Both stevioside and rebaudioside represent $90 \%$ by weight of all the sweet glycosides present in the leaves and have a structure with an aglycone in common: steviol (ent-13-hydroxykaur-16-en-18-oic acid), which distinguishes both molecules is the glycosylation pattern. ${ }^{4}$ The configuration of steviol was deciphered more than 30 years ago, and it has been concluded that steviol is synthesized from kaurene, through the mevalonate route. ${ }^{5}$ The predominant derivatives, dulcoside A and stevioside, are used to make high potency sweeteners. Stevioside differs from rebaudioside A by having one less glucose molecule. ${ }^{6}$ In addition to stevioside and rebaudioside A, other less abundant sweet compounds such as steviolbioside, rebaudioside B, C, D, E, F, and Dulcoside A have been isolated from the $S$. rebaudiana Bertoni leaf. ${ }^{7}$ All of these isolated diterpenoid glycosides have the same chemical structure of steviol but differ in the carbohydrate residues at positions $\mathrm{C} 13$ and C19. ${ }^{8}$ Stevioside constitutes the bulk of the sweetener $(60-70 \%$ of the total) and can be up to 300 times sweeter than sugar; this compound is also responsible for the astringent taste. The rebaudioside A represents $30-40 \%$ of the total and has the sweetest taste, which is $180-400$ times sweeter than sucrose, with no bitter aftertaste. ${ }^{2}$

\section{Caloric content of steviosides}

It has been shown that the human body does not absorb stevioside by the oral route and none of the digestive enzymes of the gastrointestinal tract can degrade stevioside in its aglycone: steviol. This also happens in some animals such as pigs and rabbits. It is important to emphasize that bacteria isolated from the human colon are capable of transforming stevioside to steviol in vitro, but this has never been proved in vivo, nor that steviol, if it is ever formed, can be metabolized once produced in the colon. ${ }^{9}$ Other studies on the human digestive tract show that steviol does not change at high or low concentrations 
as observed through human feces, indicating that steviol is, in fact, the final product of stevia metabolism..$^{10}$ The study also showed that most of the steviol glycosides are absorbed and glucuronidated in the liver. The newly attached glucuronide is released into the blood and filtered through the kidneys into the urine. The small amounts of glucuronidate that remain in the colon are excreted through fecal matter, which is why steviosides are not absorbed by the human body and, therefore, are not a source of caloric energy. ${ }^{11}$

Several tests of digestion and absorption have been performed with stevioside compounds and the effect of gastric juices and digestive enzymes on them show their inability to degrade or rearrange the compounds. ${ }^{12}$ The in vitro digestibility of steviosides by various digestive enzymes has been examined for many years; for example, Hutapea found that none of the enzymes present in the digestive tract digested stevioside and in the intestinal microbiota it was hydrolyzed to both steviol and steviol-16, 17 alpha-epoxide. Later, steviol 16, 17 alpha-epoxide was again converted to steviol, which was excreted from the body in the urine as steviol glucuronide. ${ }^{8}$ This also explains why $S$. rebaudiana does not contribute calories to the human body.

\section{Effect of the sweet taste of stevia on human receptors}

In general, we found that glycosides with highly branched glucose chains at $\mathrm{C}-13$ are sweeter than unbranched glucose chains. Also, the presence of a glycosyl bond in C-19 increases the intensity of the sweetness. In 2012, Hellfritsch and colleagues investigated the potency of specific steviol glycosides to stimulate the human sweet taste receptor in vitro in HEK293 FlpIn T-Rex cells that express TAS1R2 / TAS1R3 (sweet taste receptors in humans). Hellfritsch et al. ${ }^{13}$ reported that all the steviol glycosides stimulated the functionally expressed sweet taste receptors and the scores matched those of their sensory classifications.

\section{Extraction, quantification, and identification of steviosides}

Many protocols have been developed for the extraction of steviol glycosides from plant material. Most of them start with an extraction based on water or alcohol, followed by several purification steps using, for example, pigment removal agents, partitioning procedures (which often involve the extraction of steviol glycosides from the aqueous phase with n-butanol), phase extraction, and column chromatography. The application of supercritical fluid extraction and nanofiltration for the extraction of steviol glycosides still requires optimization to obtain higher purification levels or yields. Specific methods have also been developed for the extraction of steviol glycosides or their metabolites from blood, urine, and feces, as well as food. ${ }^{14}$ The classic techniques used by manufacturers for the extraction of glycosides include maceration and thermal extraction. To increase the yield and quality of the extracted products, several intensification techniques have been developed such as ultrasonic waves, supercritical fluids, and microwaves associated with the extraction of plant compounds. One of the most promising advances in this regard took place in 2000 , when Zhang et al., ${ }^{15}$ developed a multilayer membrane process with the ability to concentrate sweeteners from glycosides on a large scale (tons, even), with the additional benefit that components responsible for the bitter taste are eliminated in the process. ${ }^{15}$

At an industrial level, manufacturers usually use the same basic steps and the same methodology that involves extraction, purification, and separation to extract the steviol glycosides from the leaves, although there are variations in different stages of the process that involve purification and separation of the glycosides. The commercial products contain, in their majority, around $90 \%$ of steviol glycosides in general; as well as $80 \%$ of the two main glycosides ${ }^{16}{ }^{1}$ For their quantification, steviol glycosides have been purified by extensive recrystallization, often together with preparative RP-HPLC to obtain highly pure compounds. ${ }^{14}$ Another option to determine the concentration of steviosides is exemplified by the methodology adapted by Jiménez et al., ${ }^{17}$ which involves separating glycosidic compounds (after aqueous extraction) according to their molecular weight through an amino column using high-performance liquid chromatography, resolution. The results are expressed in percentages and are compared with standards. The procedures for the isolation of stevioside from $S$. rebaudiana leaves at pilot scale involve mainly liquid extraction with solvents such as chloroform-methane, glycerol, and propylene glycol, followed by a refinement that involves extraction in a polar organic solvent, discoloration, coagulation, ion exchange, chromatography and crystallization. ${ }^{18}$

\section{Safety in stevioside consumption}

Although refined leaf extracts or pure diterpene glycosides of $S$. rebaudiana are widely used as sucrose substitutes in some countries, in others, a more restrictive approach has been adopted. In the US, rebaudioside A and highly purified steviol glycosides are considered GRAS (Generally Recognized As Safe); this is because some studies have reported adverse effects in murine models. Previous work has reported that, although steviol glycosides are not mutagenic, the aglycone of steviosides, steviol, exhibited a mutagenic potential in the presence of a specific metabolic activation system. However, subsequent studies showed that neither steviol nor steviol glycosides are absorbed by humans. The glycosides can be hydrolyzed by the gut microbiota to steviol, but high levels of steviol glycosides or other related compounds are not detected in the blood or urine of human subjects. Esteviol glucuronide is detected in plasma and excreted in urine and stool. Because most of the steviosides are not metabolized, it can be stated that steviol glycosides are not genotoxic or carcinogenic and that they do not induce any reproductive or developmental toxicity. Geuns et al., ${ }^{19}$ showed that both steviol and its metabolites do not accumulate in the human body, although we have greater safety in healthy subjects, in whom doses of up to $750 \mathrm{mg}$ /day have been tested without harmful effects on blood pressure or insulin levels. On the other hand, in 2005, the scientific committee of the European Food Safety Authority (EFSA) issued an opinion supported by its scientific panel and declared that, after a thorough review, the Admissible Daily Intake (ADI) of stevia was established at $4 \mathrm{mg} / \mathrm{kg}$ of body weight/day. Subsequently, the same body declared having reassessed the safety of steviol glycosides on different occasions (2000, 2005, 2006, 2007, and 2009) and concluded that there was no objection to maintain the $\mathrm{ADI}$ in the values previously established. ${ }^{16}$

\section{Effects of steviosides consumption on human health}

About the characteristics of $S$. rebaudiana beyond its sweetening potential, several studies have been published where antimicrobial, antifungal, hepatoprotective, hypoglycaemic (aqueous extract), antitumor, anti-rotavirus, anti-HIV, antihypertensive, antiviral and other effects are attributed to it. Other popular applications of stevia and stevioside (mainly in Latin America and the East) include stimulation of alertness and as a supplement against fatigue; it is also attributed an improvement in the process of digestion and other gastrointestinal functions; in addition to regulating blood glucose 
levels, help in the recovery of liver, pancreas and spleen. ${ }^{1}$ Many of these effects are attributed to the phenolic compounds present in the plant (in the leaves and, to a lesser extent in the stem), said compounds are commonly found in both edible and inedible plants. They are important in the plant for the normal development of growth and defense against the attack of bacteria, parasites, infections, and injuries. Also, the presence of these compounds in injured plants can have an important effect on oxidative stability and microbial safety. Although phenolic compounds have no known nutritional function, they can be important for human health because of their antioxidant potential. $^{20}$

\section{Main beneficial effects on health}

\section{Antiobesogenic effect}

As regards the ability of $S$. rebaudiana to contribute to the treatment of obesity, it has been demonstrated in murine models that supplementation with an extract of the plant can promote weight loss, as is the case of the study by Park et al. ${ }^{21}$ They found that supplementation with Stevia rebaudiana significantly decreased body fat weight, especially epididymal fat in a murine model with c57bL/6 mice fed a diet high in fat and sucrose.

In this study, it was also recorded that the concentrations of triglycerides were significantly lower in both serum and liver in the groups supplemented with stevia. However, there were no significant effects on serum concentrations of HDL cholesterol or total cholesterol. Additionally, other researchers have reported a significantly reduced concentration of serum triglycerides, as well as total cholesterol and LDL cholesterol in diabetic and hypertensive patients supplemented with stevia extract. ${ }^{22}$ Some consumers of stevia and steviosides reported a decrease in the desire for sweets and fatty foods after consumption. Some users have reported that the consumption of improved stevia tea helped reduce their desire for tobacco and alcoholic beverages.

\section{Antitumoral effect}

The extracts of stevia leaves and the presence of polyphenolic constituents have shown an inhibitory effect on the initiation and promotion of tumors. It is known that stevioside, isoesteviol, steviol, follicular aglycones, and other metabolites inhibit tumor formation in several ways: by blocking the early antigen of Epstein-Barr virus, induction and also by reducing the production of tumors in the model of carcinogenesis of the mouse skin in two stages after exposure to 7,12 dimetibenz [a] anthracene and 12-O-tetradecanoylphorbol-13acetate. ${ }^{23} \mathrm{~A}$ compound of diterpenic nature present in the extract of the stevia leaf known as Labdane sclareol has antitumor and cytotoxic properties. ${ }^{24}$ Studies have shown the inhibitory effects of extracts and their polyphenolic components in the promotion and initiation of tumors. In the mouse skin carcinogenesis model in two stages after sequential exposure to 7,12-dimethylbenz [a] anthracene (DMBA) and 12-O-tetradecanoylphorbol-13-acetate (TPA). ${ }^{25}$

\section{Anti-diabetes effect}

The consumption of stevia leaf is not only considered safe for people with diabetes but it has been shown that it can help in the treatment of the disease. In 2011, Mishra and his team replaced the sucrose consumed in coffee, tea or milk with a stevia extract to 16 people diagnosed with diabetes. After 15 days, fasting and postprandial glucose values were taken and a significant decrease was found in both parameters in the groups in which the substitution was carried out. ${ }^{1}$ According to clinical studies, the traditional use of stevia extract increases insulin secretion and insulin sensitivity. The improvement in the latter is influenced by the components of the stevia leaves and could be linked to the inhibited hepatic expression of PEP carboxykinase and gluconeogenesis together with the stimulation of hepatic glycogen synthesis. In mice, it has been shown that cells isolated from mouse pancreatic islets have shown an increase in insulin production by the action of rebaudioside A. It is also known that stevioside promotes the secretion of insulin activated by glucose, without affecting fasting insulinemia. The evidence supports the healthy glucoregulation activity of stevioside. ${ }^{26}$

The stevia leaf, the powder and its extract of polyphenols have also been shown to exert a significant decrease in the levels of liver enzymes (ALT and AST), as well as a protector of the liver damage of rats treated with diabetes. In the study, the possibility was considered that reduced levels of ALT and AST sera by stevia were an indication of a possible reversal of plasma membrane damage produced by diabetes. In 2000, Jeppesen and colleagues published a study stating that stevia intake caused a slight suppression of plasma glucose levels and significantly increased glucose tolerance in healthy human adults. Steviol glycosides proved to have an enhancing effect on insulin secretion by acting directly on the $\beta$ cells without altering the activity of the K + ATP channel and the level of cAMP in the islets, thus documenting stevioside and steviol as potent antihyperglycemic agents. ${ }^{27}$ Stevioside has also been characterized by regulating blood glucose levels by increasing not only insulin secretion but also the use of insulin in rats with induced lack of insulin. This phenomenon is related to the decrease of the gene expression of phosphoenolpyruvate carboxykinase (PEPCK) in rat liver by the action of stevioside by decreasing the gluconeogenesis suggested by Chen. ${ }^{28}$

\section{Anticariogenic activity}

It has been reported that a greater amount of stevioside and Stevia extract can reduce bacterial growth. The required concentration of stevioside as a sweetener is quite low compared to sugar. Therefore, stevioside can be a substitute for the cariogenic compounds present in sucrose. Several studies have concluded that stevioside and rebaudioside A are non-cariogenic sweetener. ${ }^{29}$ It has also been shown that stevioside inhibits the growth and reproduction of some bacteria responsible for dental caries.

\section{Hypotensive activity}

Steviosides are capable of inducing diuresis, as well as vasorelaxation and natriuresis, which leads to a decrease in plasma volume. Some studies based on human beings have also suggested the role of stevioside in the cardiovascular system and as a cause of hypotension that reduces the duration of systole, which could reduce the likelihood of suffering a stroke. Long-term clinical trials of stevioside in humans have indicated that their continued use may reduce systolic and diastolic blood pressure, although no significant side effects on lipids or fasting glucose were observed. ${ }^{23}$ The glycosides of steviol and steviol belong to diterpenes and have a structure similar to that of steroids. Recent data showed that stevioside and steviol exert effects mediated by the progesterone receptor and act as glucocorticoid agonists. ${ }^{30}$

\section{Effects on blood pressure and kidney function}

Different research reports based on the effects of stevioside have affirmed variable effects on renal function and regulation of blood 
pressure. It decreases the mean arterial pressure together with the decrease in renal vascular resistance, produces diuresis and increases the fractional excretion of $\mathrm{Na}+$ and $\mathrm{K}+$. The lack of effect on the glomerular filtration rate implies that stevioside has a vasodilation effect on afferent and efferent arterioles. ${ }^{23}$

\section{Anti-inflammatory effect}

It has been found that steviosides can attenuate the synthesis of inflammatory mediators in THP-1 cells stimulated by lipopolysaccharide (LPS) interfering with Kappa B kinases and their signaling pathway, so they can be considered beneficial compounds with anti-inflammatory action and immunomodulatory. ${ }^{31}$ Anti- inflammatory effects of steviol and stevioside have also been observed in epithelial cells of the colon. It has been shown that stevioside exhibits inhibitory effects on the contraction of the smooth muscle of the intestine, whose inhibition is related to diarrhea associated with hypermotility. ${ }^{32}$

\section{The optimum dose of Stevia rebaudiana Bertoni}

Although the information regarding the reported effects of $S$. rebaudiana is vast. So far there is no consensus regarding the adequate dose to achieve the desired effects for the different documented benefits. A compilation in the consulted bibliography is presented in Table 1.

Table I Reported dosage of S. rebaudiana Bertoni in clinical trails

\begin{tabular}{|c|c|c|c|c|}
\hline Authors, year & Dose & Subjects & Time & Results \\
\hline $\begin{array}{l}\text { Gregersen et } \\
\text { al., } 2004\end{array}$ & $1,000 \mathrm{mg}$ of stevioside. & Diabetic human & Single dose & $\begin{array}{l}\text { S. rebaudiana was able to reduce the postprandial area under the cur } \\
\text { of glucose by } 18 \% \text { relative to control ( } 1 \mathrm{~g} \text { corn starch) and appeared } \\
\text { the insulin:glucose ratio in serum by } 40 \% \text {. }\end{array}$ \\
\hline $\begin{array}{l}\text { Geuns et al., } \\
2007\end{array}$ & $250 \mathrm{mg}$ of stevioside (capsules). & 10 healthy subjects & $\begin{array}{l}\text { Twice dailyl } \\
3 \text { days }\end{array}$ & $\begin{array}{l}\text { No significant difference was found for mean blood glucose ar } \\
\text { between control and stevioside conditions. }\end{array}$ \\
\hline $\begin{array}{l}\text { Maki et al., } \\
2008\end{array}$ & $1000 \mathrm{mg} /$ day of stevioside. & $\begin{array}{l}\text { Men/Women } \\
\text { Diabetes Type } 2\end{array}$ & 16 weeks & $\begin{array}{l}\text { The supplementation did not affect glucose homeostasis/restil } \\
\text { pressure. }\end{array}$ \\
\hline $\begin{array}{l}\text { Geeraert, } \\
2010\end{array}$ & $\begin{array}{l}\text { Stevioside dissolved in } \\
\text { physiological } \\
\text { saline solution }(0.9 \% \quad \mathrm{NaCl}) \\
(1 \mathrm{mg} / \mathrm{ml}) \text { and administered orally } \\
\text { at a dose of } 10 \mathrm{mgkg} / \text { day. }\end{array}$ & $\begin{array}{l}\text { Twelve-week-old mice treated with } \\
\text { stevioside }(10 \quad \mathrm{mg} / \mathrm{kg}, \quad n=14) \text { or } \\
\text { placebo }(n=20) \text {. }\end{array}$ & 12 weeks & $\begin{array}{l}\text { Stevioside had no effect on weight and triglycerides, but lowered glu } \\
\text { insulin. This treatment improved adipose tissue maturation, and i } \\
\text { glucose transport, insulin signaling and antioxidant defense in whitt } \\
\text { adipose tissues. Stevioside treatment also was associated with } \\
\text { insulin signaling and antioxidant defense in both the adipose tissut } \\
\text { vascular wall. }\end{array}$ \\
\hline
\end{tabular}

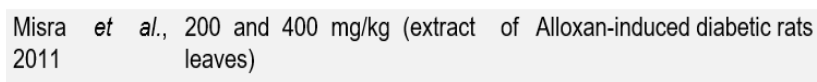

10 days

The supplementation produced a delayed but significant $(p<0.01)$ de the blood glucose level, without hypoglycemia. Stevia rebaudian extract was found to antagonize the necrotic action of alloxan and th re-vitalizing effect on $\beta$-cells of the pancreas.

250 and $500 \mathrm{mg} / \mathrm{kg} / \mathrm{d}$ decreases the omentin level indirectly via insulin sensitivity and lowering blood glucose in STZ-induced diabetic

Stevia rebaudiana Bertoni supplementation has a potential hypoglycer Along with an anti-hypertensive effect. Long term studies on a sizabl are necessary. (age 35-55)

Ahmad et al., 200. 300, 400 y 500 ppm $/ \mathrm{kg}$. Streptozotocin-induced diabetic rats 8 weeks

2018 Stevia rebaudiana Bertoni aqueous extract.

\section{Use of steviosides in the food industry}

Steviol glycosides are thermally stable, which makes them more suitable for use in cooked foods and beverages. Stevia extracts and steviosides are mainly used as non-caloric sweeteners and flavor enhancers in a variety of food and beverage products, such as tea, coffee, soft drinks, weight-loss diets or diabetic patients and juices fruits. As stevia sweeteners are heat stable and non-fermented, they are used in a wide range of products including baked and cooked foods. It has also been used as a source of antioxidants and as an enhancer of alcoholic beverages (aging agent and catalyst). ${ }^{34}$ The steviol glycoside preparations are white or slightly yellowish, crystalline and odorless powders; these glycosides are freely soluble in water and ethanol and can be easily extracted with an aqueous solvent. The compounds are exceptionally stable at $\mathrm{pH}$ values ranging from 2 to 10 . In acid solutions, stevioside is highly stable and does not interact with other food components or cause browning. Stevia sweeteners can withstand temperatures of up to $200^{\circ} \mathrm{C}$, unlike sugar, which begins to caramelize at about $150-160^{\circ} \mathrm{C}$. Even the sweetness of aqueous stevioside solutions does not change when heated at $95^{\circ} \mathrm{C}$ for 2 hours..$^{35}$ The sweetness of stevioside is superior to sugar in the softness, freshness sensation, and length of time of permanence. The stability of steviol glycosides has also been evaluated in various food products such as semi-skimmed milk, soy drinks, fermented milk drinks, ice cream, yogurt (made with whole cream and skimmed milk), dried biscuits and jam. Storage conditions were recommended for each type of food $\left(-18{ }^{\circ} \mathrm{C}\right.$ for ice cream, $6^{\circ} \mathrm{C}$ for yogurt and $20^{\circ} \mathrm{C}$ for skimmed and 
fermented milk). The recovery of steviosides was $96 \%$, which shows that the steviol glycosides had not decomposed in any of the samples analyzed..$^{34}$ Mogran \& Dashora ${ }^{36}$ determined in 2009 the amount of stevia extract (obtained by boiling stevia leaf powder in water) and other commercial sweeteners needed to produce a degree of sweetness equivalent to sugar. Once the quantities required to reach the desired sweetness were determined, various products were elaborated among which were: milk, coffee, tea, milkshake, curd, lemon water, custard, replacing the sugar in the recipes with the stevia extract. The results revealed that $1.5 \mathrm{ml}$ of stevia extract in $100 \mathrm{~mL}$ of liquid was equivalent to $5 \mathrm{~g}$ of sugar.

Despite these characteristics, the bitter taste of rebaudioside A limits its application in food and pharmaceutical products. Several attempts have been made to overcome this by modifying stevioside in an intermolecular transglycosylation reaction, catalyzed by several enzymes, during which other carbohydrates are bound at positions $\mathrm{C} 13$ and $\mathrm{C} 19 .{ }^{23}$

Many products sweetened with stevia extract tend to have a bitter residual taste. This can be attributed to the high proportions of stevioside glucoside contained in the mixture of leaf components obtained during the extraction process. This negative element is eliminated when plant varieties with higher concentrations of rebaudioside A are used, or simply by using steviol glycosides in pure form, due to their superior taste profile. ${ }^{37}$

\section{Commercial presentations}

Currently, there is a significant variety of commercial presentations. It is usually found as cartons similar to sugar, such as bottles with extract to sweeten or as a powder of the Stevia leaf; in some of these presentations, especially in the powder versions, other sweeteners are often added such as sorbitol, sucralose or even sucrose, to name a few. It is important to remember that the beneficial health effects described above are attributed only to leaves, extracts or steviosides alone; so far no report has proven health effects in commercial presentations.

\section{Conclusions}

Stevia rebaudiana Bertoni is a plant of diterpenic nature whose potency is superior to that of other sweeteners such as sucrose, due to the variety of glycosides synthesizes on the leaves. These glycosides and other compounds present in S. rebaudiana leaves have a wide therapeutic potential in degenerative diseases, especially in diabetes. As well have shown antihyperglycemic, antihypertensive, antioxidant, anticarcinogenic, anti-inflammatory, anti-cariogenic properties. $S$. rebaudiana represents a natural and healthy alternative to other sweeteners. However, research is still needed to expand knowledge and potential application, especially in humans, since some of these benefits have only been documented in high doses, not in those that are generally consumed as a conventional sweetener. It is hoped that in the future, the research can be scaled up to further tests in humans to corroborate the effects and use stevia and its by-products as a natural and affordable alternative to conventional treatments.

\section{Acknowledgements}

MESP and RME thank to Instituto Politécnico Nacional, COFAA, EDI and SNI. KNEL thanks the scholarship of the CONACyT.

\section{Conflicts of interest}

All authors declare no conflict of interest.

\section{Funding}

None.

\section{References}

1. Misra H, Soni M, Silawat N, et al. Antidiabetic activity of medium-polar extract from the leaves of Stevia rebaudiana Bert. (Bertoni) on alloxaninduced diabetic rats. Journal of Pharmacy and Bioallied Sciences. 2011;3(2):242.

2. Tavarini S, Angelini LG. Stevia rebaudiana Bertoni as a source of bioactive compounds: the effect of harvest time, experimental site and crop age on steviol glycoside content and antioxidant properties. Journal of the Science of Food and Agriculture. 2013;93(9):2121-2129.

3. Yadav AK, Singh S, Dhyani D, et al. A review of the improvement of stevia [Stevia rebaudiana (Bertoni)]. Canadian Journal of Plant Science. 2011;91(1):1-27.

4. Philippaert K, Pironet A, Mesuere M, et al. Steviol glycosides enhance pancreatic beta-cell function and taste sensation by the potentiation of TRPM5 channel activity. Nature communications. 2017;8:14733.

5. Brandle JE, Telmer PG. Steviol glycoside biosynthesis. Phytochemistry. 2007;68(14):1855-1863.

6. Shivanna N, Naika M, Khanum F, et al. Antioxidant, anti-diabetic and renal protective properties of Stevia rebaudiana. Journal of Diabetes and its Complications. 2013;27(2):103-113.

7. Pawar RS, Krynitsky AJ, Rader JI. Sweeteners from plants-with emphasis on Stevia rebaudiana (Bertoni) and Siraitia grosvenorii (Swingle). Analytical and bioanalytical chemistry. 2013;405(13):43974407.

8. Chatsudthipong V, Muanprasat C. Stevioside and related compounds: therapeutic benefits beyond sweetness. Pharmacology \& Therapeutics. 2009;121(1):41-54

9. Geuns JM. Stevioside. Phytochemistry. 2003;64(5):913-921.

10. Koyama E, Sakai N, Ohori Y, et al. Absorption and metabolism of glycosidic sweeteners of stevia mixture and their aglycone, steviol, in rats and humans. Food and chemical toxicology. 2003;41(6):875-883.

11. Gupta E, Purwar S, Sundaram S, et al. Nutritional and therapeutic values of Stevia rebaudiana: A review. Journal of Medicinal Plants Research. 2013;7(46):3343-3353.

12. Wingard RE, Brown JP, Enderlin FE, et al. Intestinal degradation and absorption of the glycosidic sweeteners stevioside and rebaudioside A. Experientia. 1980;36(5):519-520.

13. Hellfritsch C, Brockhoff A, Stähler F, et al. Human psychometric and taste receptor responses to steviol glycosides. Journal of agricultural and foo d chemistry. 2012;60(27):6782-6793.

14. Ceunen S, Geuns JM. Steviol glycosides: chemical diversity, metabolism, and function. Journal of natural products. 2013;76(6):1201-1228.

15. Zhang SQ, Kumar A, Kutowy O. Membrane-based separation scheme for processing sweeteners from stevia leaves. Food Research International. 2000;33(7):617-620

16. EFSA Panel on Food Additives and Nutrient Sources added to Food (ANS). Scientific Opinion on the safety of steviol glycosides for the proposed uses as a food additive. EFSA Journal. 2010;8(4):1537. 
17. Jiménez T, Cabrera G, Álvarez E, et al. Evaluation of the content of stevioside and rebaudioside A in a population of Stevia rebaudiana Bertoni (kaâ heê) commercially cultivated. A preliminary study. Memorias del Instituto de Investigaciones en Ciencias de la Salud. 2010;8(1):47-53.

18. Puri M, Sharma D, Tiwari AK. Downstream processing of stevioside and its potential applications. Biotechnol Adv. 2011;29(6):781-791.

19. Geuns JM, Buyse J, Vankeirsbilck A, et al. Metabolism of stevioside by healthy subjects. Experimental biology and medicine. 2007;232(1):164173.

20. Tadhani MB, Patel VH, Subhash R. In vitro antioxidant activities of Stevia rebaudiana leaves and callus. Journal of Food Composition and Analysis. 2007;20(3-4):323-329.

21. Park JE, Cha YS. Stevia rebaudiana Bertoni extract supplementation improves lipid and carnitine profiles in C57BL/6J mice fed a high-fat diet. Journal of the Science of Food and Agriculture. 2010;90(7):1099_ 1105 .

22. Savita SM, Sheela K, Sunanda S, et al. Health implications of Stevia rebaudiana. Journal of Human Ecology. 2004;15(3):191-194.

23. Mathur S, Bulchandani N, Parihar S, et al. A critical review on steviol glycosides: pharmacological, toxicological and therapeutic aspects of high potency zero caloric sweetener. International Journal of Pharmacology. 2017;13(7):916-928.

24. Kaushik R, Narayanan P, Vasudevan V, et al. Nutrient composition of cultivated stevia leaves and the influence of polyphenols and plant pigments on sensory and antioxidant properties of leaf extracts. Journal of food science and technology. 2010;47(1):27-33.

25. Konoshima T, Takasaki M. et al. Cancer-chemopreventive effects of natural sweeteners and related compounds. Pure and applied chemistry. 2002;74(7):1309-1316.

26. Gregersen S, Jeppesen PB, Holst JJ, et al. Antihyperglycemic effects of stevioside in type 2 diabetic subjects. Metabolism. 2004;53(1):73-76.

27. Jeppesen PB, Gregersen S, Poulsen CR, et al. Stevioside acts directly on pancreatic $\beta$ cells to secrete insulin: Actions independent of cyclic adenosine monophosphate and adenosine triphosphate-sensitivie $\mathrm{K}+-$ channel activity. Metabolism. 2000;49(2):208-214.
28. Chen TH, Chen SC, Chan P, et al. Mechanism of the hypoglycemic effect of stevioside, a glycoside of Stevia rebaudiana. Planta médica. 2005;71(02):108-113.

29. Yadav SK, Guleria P. Steviol glycosides from Stevia: biosynthesis pathway review and their application in foods and medicine. Critical reviews in food science and nutrition. 2012;52(11):988-998.

30. Shannon M, Rehfeld A, Frizzell C, et al. In vitro bioassay investigations of the endocrine-disrupting potential of steviol glycosides and their metabolite steviol, components of the natural sweetener Stevia. Molecular and cellular endocrinology. 2016;427:65-72.

31. Boonkaewwan C, AO M, Toskulkao C, et al. Specific immunomodulatory and secretory activities of stevioside and steviol in intestinal cells. Journal of agricultural and food chemistry. 2008;56(10):3777-3784.

32. Pariwat P, Homvisasevongsa S, Muanprasat C, et al. A natural plantderived dihydroisosteviol prevents cholera toxin-induced intestinal fluid secretion. Journal of Pharmacology and Experimental Therapeutics. 2007;324(2):798-805.

33. Kroyer G. Stevioside and Stevia-sweetener in food: application, stability, and interaction with food ingredients. Journal für Verbraucherschutz und Lebensmittelsicherheiit. 2010;5(2):225-229.

34. Abou-Arab AE, Abou-Arab AA, Abu-Salem MF. Physico-chemical assessment of natural sweeteners steviosides produced from Stevia rebaudiana Bertoni plant. African Journal of Food Science. 2010;4(5):269-281.

35. Jooken E, Amery R, Struyf T, et al. Stability of steviol glycosides in several food matrices. Journal of agricultural and food chemistry. 2012;60(42):10606-10612.

36. Mogra R, Dashora V. Exploring the use of Stevia rebaudiana as a sweetener in comparison with other sweeteners. Journal of Human Ecology. 2009;25(2):117-120.

37. Dacome AS, Da Silva CC, Da Costa CE, et al. Sweet diterpenic glycosides balance of a new cultivar of Stevia rebaudiana (Bert.) Bertoni: Isolation and quantitative distribution by chromatographic, spectroscopic, and electrophoretic methods. Process Biochemistry. 2005;40(11):3587-594. 